\title{
EXPERIMENTAL AND NUMERICAL ANALYSIS OF THE TENSILE TEST USING SHEET SPECIMENS
}

\author{
Eduardo E. Cabezas and Diego J. Celentano \\ Departamento de Ingeniería Mecánica \\ Universidad de Santiago de Chile \\ Av. Bdo. O'Higgins 3363 \\ Santiago, Chile \\ e-mail:dcelenta@lauca.usach.cl
}

Key words: tensile test, mechanical characterization, sheet specimens, large strains, plasticity, finite element formulations

\begin{abstract}
This paper presents an experimental and numerical study of the mechanical behaviour of SAE 1045 steel sheet specimens during the conventional tensile test. Due to the complex stress state that develops at the neck for high levels of axial deformation, an experimental-numerical methodology is proposed in order to derive the elastic and hardening parameters which characterize the material response. Such methodology is essentially an extension to sheet samples of the well established procedure used for cylindrical ones. The simulation of the deformation process during the whole test is performed with a finite element large strain elastoplasticity-based formulation. Finally, the experimental validation of the obtained numerical results allows to assess the performance of the proposed methodology for the $3 D$ analysis of sheet specimens and, in addition, to discuss the range of applicability of plane stress conditions.
\end{abstract}




\section{INTRODUCTION}

The tensile test is an important standard engineering procedure useful to characterize some relevant elastic and plastic variables related to the mechanical behaviour of materials. Due to the non-uniform stress and strain distributions existing at the neck for high levels of axial deformation, it has been long recognized that significant changes in the geometric configuration of the specimen have to be considered in order to properly describe the material response during the whole deformation process up to the fracture stage.

Although in many engineering applications the design of structural parts is restricted to the elastic response of the materials involved, the knowledge of their behaviour beyond the elastic limit is relevant since plastic effects with usually large deformations take place in the previous manufacturing procedures such as forming, forging, etc.

The diffused necking process of both cylindrical and sheet samples used in the tensile test has been extensively studied (see e.g. Nádai, 1950 and Bridgman, 1952). In particular, Bridgman (1952) derived, based on some geometric considerations of the deformation pattern, analytical expressions for the stress distribution at the neck written in terms of the ratio $\chi / a$ at the necking zone ( $\chi$ is the curvature radius of the neck and $a$ is either its diameter $D$ or its width $w$ for the cylindrical or sheet samples, respectively; see Fig. 1). As $\chi$ is an extremely difficult variable to be measured in practice, alternative equations depending only on $a$ have been also proposed exclusively for cylindrical samples (Bridgman, 1952). Therefore, the applicability of this methodology to flat bars is rather limited. However, it should be noted that the use of sheet specimens is the unique possibility to test different strip-manufactured materials as those produced by means of rolling processes.

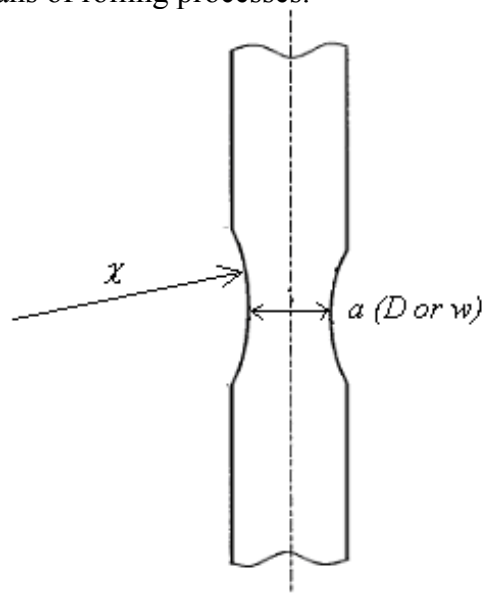

Figure 1. Analysis of a SAE 1045 steel tension specimen: schematic representation of the necking zone for both cylindrical and sheet specimens.

In recent years, several finite element large strain formulations usually defined within the plasticity framework have been developed and applied to the analysis of this test under isothermal and non- 
isothermal conditions (see e.g. Wriggers et al., 1989; Simo and Armero, 1992; Armero and Simo, 1993; García Garino and Oliver, 1993; Simo, 1995; Goicolea et al., 1996 and references therein). Moreover, some of such formulations have been validated, generally under isothermal conditions, with experimental data considering cylindrical specimens of different materials. In contrast, there have been only few studies focused on the necking phenomenon of strips (see e.g. Tvergaard, 1993 and Ling, 1996).

The aim of this paper is to present an experimental analysis and a numerical simulation of the mechanical behaviour during the tensile test experienced by sheet specimens of SAE1045 steel. The experimental procedure undertaken to characterize some specific features of the material response is described in Section 2. In particular, details on the derivation of the parameters involved in the assumed exponential plastic hardening law are given. Moreover, the governing equations together with the constitutive model proposed to simulate the deformation process that takes place during the test are presented in Section 3. This large strain isotropic elastoplasticity-based formulation includes the definitions of a specific free energy function and plastic evolution equations which are the basis to derive the stress-strain relationship and a thermodynamically consistent expression for the internal dissipation. The corresponding finite element model is briefly presented in Section 4 where a particular treatment of the incompressible plastic flow in order to overcome the well-known volumetric locking in the numerical behaviour is discussed. It should be mentioned that this finite element formulation is an alternative approach to existing methodologies dealing with large plastic deformations.

The numerical simulation of the tensile test applied to cylindrical and sheet specimens of SAE1045 steel is performed in Section 5. The results obtained with the proposed formulation are validated with the corresponding experimental measurements. Aside from the engineering stress-strain curve, different results at the section undergoing extreme necking are specifically analysed: ratio of current to initial diameter in terms of the elongation and both load and mean true axial stress versus logarithmic strain. Furthermore, computed non-uniform stress components and effective plastic deformation contours at the fracture stage are also shown confirming the strong influence of the necking formation in the material response.

\section{EXPERIMENTAL PROCEDURE}

The experimental procedure adopted in this work to characterize the mechanical behaviour of a material consisted in the following steps:

1) Selection of the material and the specimens to be tested according to the ASTM standards (Annual Book of ASTM Standards, 1988). The chosen material is SAE 1045 steel considering cylindrical and sheet specimens as sketched in Fig. 2. The distance between the two black markers denotes the initial extensometer length taken as $50 \mathrm{~mm}$ for both cases. A nearly linear gradual reduction in diameter and width (for the cylindrical and sheet specimens, respectively) is considered in order to trigger the necking development which has to take place approximately at the middle of the extensometer length. This tapered profile fits the ASTM standards since the difference between the maximum and minimum diameter or width values existing in the extensometer length is lower than $1 \%$. 
a)

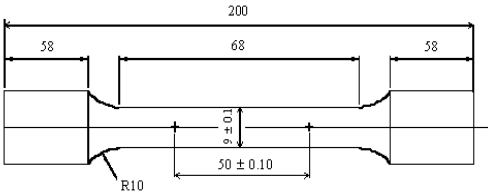

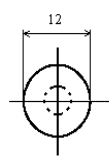

b)

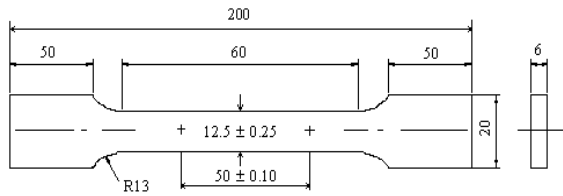

Figure 2. Analysis of a SAE 1045 steel tension specimen: geometric configurations for a) cylindrical and b) sheet samples (dimensions in $\mathrm{mm}$, initial extensometer length=50 $\mathrm{mm}$ and load cell speed of 2.5 $\mathrm{mm} / \mathrm{min}$ for both cases).

2) Chemical characterization to check an adequate composition according to the selected material. This routine task is carried out by means of an optical spectrometer. The average chemical composition for the studied steel is shown in Table 1.

Table 1. Analysis of a SAE 1045 steel tension specimen: average chemical composition (\% in weight).

\begin{tabular}{||c||c||c||c||c||}
\hline $\mathrm{C}$ & $\mathrm{Si}$ & $\mathrm{Mn}$ & $\mathrm{P}$ & $\mathrm{S}$ \\
0.447 & 0.213 & 0.756 & 0.0148 & 0.0372 \\
\hline \hline $\mathrm{Cr}$ & $\mathrm{Mo}$ & $\mathrm{Ni}$ & $\mathrm{Al}$ & $\mathrm{Cu}$ \\
0.0635 & 0.0139 & 0.0914 & $<0.00106$ & 0.277 \\
\hline \hline $\mathrm{Nb}$ & $\mathrm{Ti}$ & $\mathrm{V}$ & $\mathrm{W}$ & $\mathrm{Pb}$ \\
$<0.0050$ & 0.00219 & 0.00917 & $<0.01$ & $<0.005$ \\
\hline \hline $\mathrm{Sn}$ & $\mathrm{B}$ & $\mathrm{Fe}$ & $\mathrm{Co}$ & \\
0.0159 & $<0.00051$ & 98.02 & 0.0172 & \\
\hline
\end{tabular}

3) Mechanical tensile test. The engineering stress-strain curves obtained with five cylindrical and sheet specimens considering a load cell speed of $2.5 \mathrm{~mm} / \mathrm{min}$ (value within the range specified by the ASTM standards) are plotted in Fig. 3. As usual, the engineering stress is defined as $P / A_{0}$, where $P$ is the axial load and $A_{0}$ is the initial transversal area while the engineering strain or elongation is computed as $\left(L-L_{0}\right) / L_{0}$, with $L$ and $L_{0}$ being the current and initial extensometer lengths, respectively. At the beginning of the deformation process the material behaves elastically. After the yield strength is reached, the plastic hardening begins and the load increases up to a maximum value for a specific elongation. Then, the load decreases since the effect of the reduction of the transversal area at the necking zone is stronger than that of the hardening mechanism. Diffuse necking has been developed for both types of samples (localized necking is only observable for very thin strips; see Ling, 1996). The effect of plastic deformation without hardening developed immediately after the elastic response (usually known as Lüders' band formation which is a typical phenomenon for steels with low to moderate carbon contents; see Dieter, 1988) can also be observed in these curves. It should be noted, however, that this fact is almost imperceptible for the test with sheet samples due to, presumably, the distorting effects produced by the machining operations necessary to make the specimens. The average experimentally for the yield strength, maximum load, maximum engineering stress and elongation at the fracture stage are summarized in Table 2. 

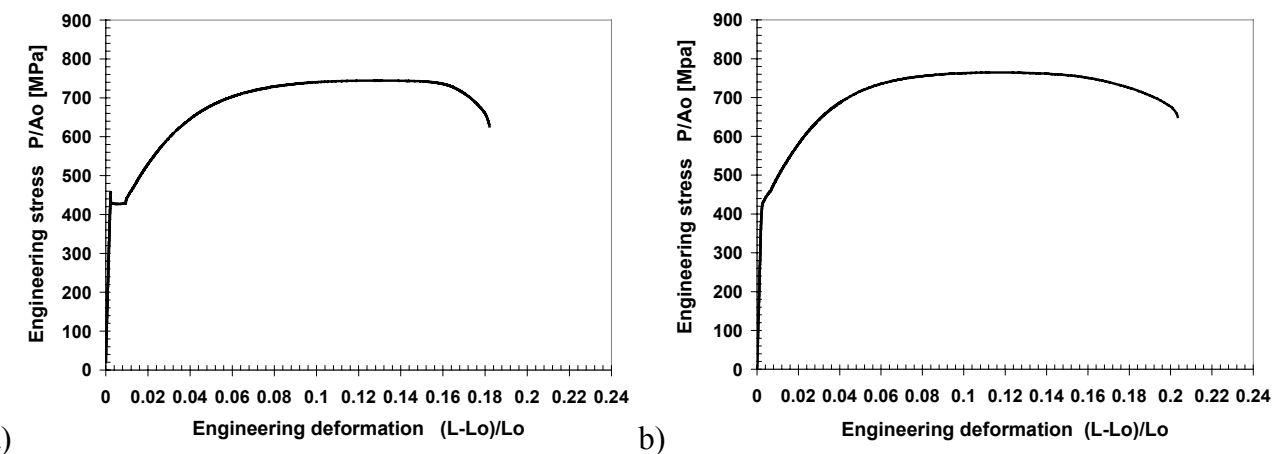

Figure 3. Analysis of a SAE 1045 steel tension specimen: average experimental stress-strain curve for a) cylindrical and $b$ ) sheet samples.

Table 2. Analysis of a SAE 1045 steel tension specimen: average experimentally measured values.

\begin{tabular}{|c||c||c||c||c||}
\hline Sample & $\begin{array}{c}\text { Yield strength } \\
\text { (elongation: 0.2\%) } \\
{[\mathrm{MPa}]}\end{array}$ & $\begin{array}{c}\text { Maximum } \\
\text { load } \\
{[\mathrm{kN}]}\end{array}$ & $\begin{array}{c}\text { Maximum } \\
\text { engineering stress } \\
{[\mathrm{MPa}]}\end{array}$ & $\begin{array}{c}\text { Elongation at the } \\
\text { fracture stage } \\
{[\%]}\end{array}$ \\
\hline \hline Cylindrical & 450.4 & 46.6 & 749.2 & 18.5 \\
\hline \hline Sheet & 451.6 & 56.5 & 762.0 & 20.0 \\
\hline
\end{tabular}

4) Characterization of the plastic behaviour. At high levels of elongation, the stress and strain distributions are no longer uniform along the specimen due to the necking formation that takes place for both cylindrical and sheet samples. Therefore, the stress-strain curve of Fig. 3 can not provide a proper description of the physical phenomena involved in the test. Following the procedure proposed by Bridgman (1952), the mechanical response can be adequately described by an alternative stress-strain curve defined in terms of the mean equivalent stress $\bar{\sigma}_{e q}$ versus an equivalent deformation $\varepsilon_{e q}$ (composed of an elastic and plastic contributions) respectively given by $\bar{\sigma}_{e q}=f_{B} P / A$ and $\varepsilon_{e q}=\bar{\sigma}_{e q} / E+\varepsilon_{p}$, where $f_{B}\left(\varepsilon_{p}\right) \leq 1$ is an assumed known correction factor applied to the mean true axial stress $P / A, A$ is the current transversal area at the necking zone $\left(A=\pi D^{2} / 4\right.$ or $A=w t$ for the cylindrical and sheet samples, respectively, where $D, w$ and $t$ are the current diameter, width and thickness of the neck), $E$ is the Young's modulus and $\varepsilon_{p}=\ln \left(A_{0} / A\right)$ is the true (logarithmic) deformation. A preliminary step to obtain such stress-strain relationship (in which, as can be seen, $D, w$ and $t$ are the additional variables to be measured), consists of deriving the $P / A-\varepsilon_{p}$ curve shown in Fig. 4 using four specimens for each of both samples.

Details of the application of this procedure are described in Sections 5.1 and 5.3 for cylindrical and sheet specimens, respectively. 

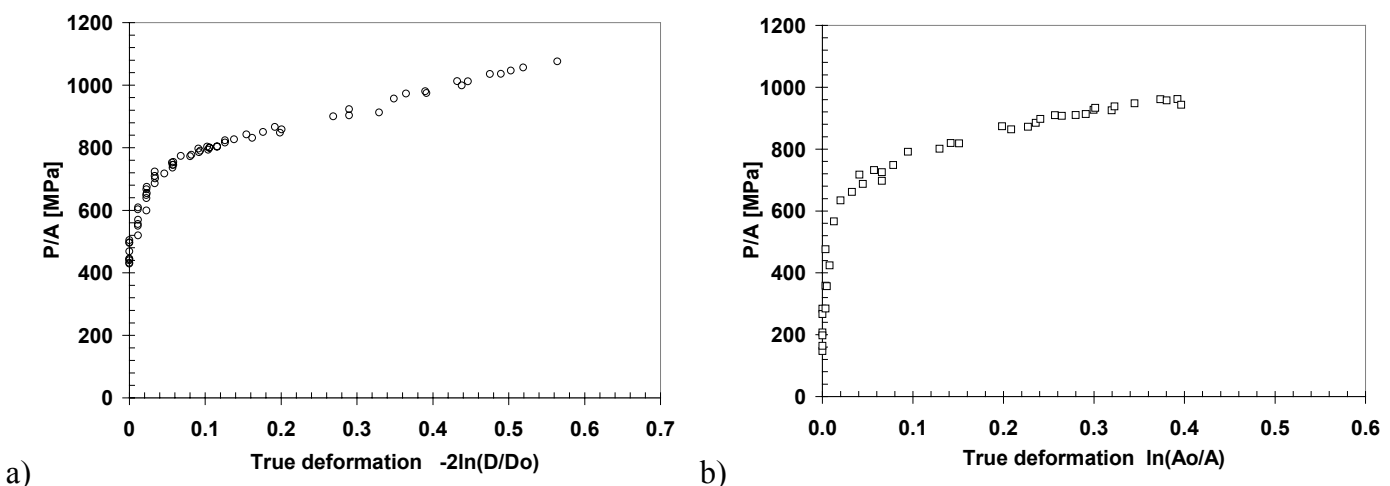

Figure 4. Analysis of a SAE 1045 steel tension specimen: mean true axial stress versus true (logarithmic) deformation for a) cylindrical and b) sheet samples.

\section{GOVERNING EQUATIONS AND CONSTITUTIVE MODEL}

The local governing equations describing the evolution of an assumed quasi-static isothermal process (i.e., that with negligible inertia effects and identically fulfilled energy balance) can be expressed by the continuity equation, the equation of motion and the dissipation inequality (all of them valid in $\Omega x \Upsilon$, where $\Omega$ is the spatial configuration of a body and $\Upsilon$ denotes the time interval of interest with $t \in \Upsilon$ ) respectively written in a Lagrangian description as (Malvern, 1969):

$\rho J=\rho_{0}$

$\nabla \cdot \sigma+\rho \mathbf{b}_{f}=\mathbf{0}$

$D_{\text {int }} \geq 0$

together with appropriate conditions and an adequate constitutive relation for the Cauchy stress tensor $\sigma$ (which is symmetric for the non polar case adopted in this work). In these equations, $\rho$ is the density, $J$ is the determinant of the deformation gradient tensor $\mathbf{F}\left(\mathbf{F}^{-1}=\mathbf{1}-\nabla \times \mathbf{u}\right.$, where $\mathbf{1}$ is the unity tensor, $\nabla$ is the spatial gradient operator and $\mathbf{u}$ is the displacement vector), the subscript 0 applied to a variable denotes its value at the initial configuration $\Omega_{0}, \mathbf{b}_{\mathrm{f}}$ is the specific body force vector and $D_{\text {int }}$ is the internal dissipation which imposes restrictions over the constitutive model definition. In this framework, a specific Helmholtz free energy function $\psi$, assumed to describe the material behaviour during the deformation process, can be defined in terms of some thermodynamic state variables chosen in this work as the Almansi strain tensor $\mathbf{e}\left(\mathbf{e}=1 / 2\left(\mathbf{1}-\mathbf{F}^{-T} \cdot \mathbf{F}^{-1}\right)\right.$, where $T$ is the transpose symbol) and a set of 
$n_{\text {int }}$ phenomenological internal variables $\alpha_{k}$ (usually governed by rate equations with $k=1, \ldots, n_{\text {int }}$ ) accounting for the non-reversible effects (García Garino and Oliver, 1993; Goicolea et al., 1996; Celentano, 2001). This free energy definition, based on the Doyle-Ericksen's approach (Doyle and Ericksen, 1956), is only valid for small elastic strains and isotropic material response, both assumptions being normally accepted for metals and other materials. Invoking the Coleman's method (Coleman and Gurtin, 1967), the following relationships are obtained: $\sigma=\rho \partial \psi / \partial \mathbf{e}$ and $D_{\text {int }}=\mathbf{q}_{k} * D \alpha_{k} / D t$ where $\mathbf{q}_{k}=-\rho_{0} \partial \psi / \partial \boldsymbol{\alpha}_{k}$ are the conjugate variables of $\alpha_{k}$ and, according to the nature of each internal variable, the symbols * and $D(\cdot) / D t$ appearing in the previous expressions respectively indicate an appropriate multiplication and a time derivative satisfying the principle of material frame-indifference (Malvern, 1969).

It is seen that the definitions of $\psi=\psi\left(\mathbf{e}, \alpha_{k}\right)$ and $D \alpha_{k} / D t$ are crucial features of the model in order to derive the constitutive equations presented above.

The internal variables and their corresponding evolution equations are defined in this work within the associate rate-independent plasticity theory context (Lubliner, 1990; Simo, 1995). A possible choice is given by the plastic Almansi strain tensor $\mathbf{e}^{\mathrm{p}}$ and the effective plastic deformation $\bar{e}^{p}$ related to the isotropic strain hardening effect (García Garino and Oliver, 1993; Goicolea et al., 1996; Celentano, 2001). The evolution equations for such plastic variables are written as:

$$
L_{v}\left(\mathbf{e}^{p}\right)=\dot{\lambda} \partial F / \partial \tau \quad \dot{e}^{p}=\dot{\lambda}
$$

where $\tau$ is the Kirchhoff stress tensor $(\tau=\mathrm{J} \sigma), L_{v}$ is the well-known Lee (frame-indifferent) derivative, $\dot{\lambda}$ is the rate (or increment in this context) of the plastic consistency parameter computed according to classical concepts of the plasticity theory (Simo, 1995) and $F=F\left(\sigma, \bar{e}^{p}\right)$ is the yield function governing the plastic behaviour of the solid such that no plastic evolutions occur when $F<0$. A Von Mises yield function is adopted:

$$
F=\sqrt{3} J_{2}-C^{p}
$$

where $J_{2}$ is the second invariant of the deviatoric part of $\tau$ and $C^{p}$ is the plastic hardening function given by:

$$
C^{p}=A^{p}\left(\bar{e}_{0}^{p}+\bar{e}^{p}\right)^{n^{p}}
$$

where $\bar{e}_{0}^{p}$ is an assumed initial value of $\bar{e}^{p}$ such that $C^{\text {th }}=A^{p} \bar{e}_{0}^{p n^{p}}$ with $C^{\text {th }}$ being the yield strength defining the material elastic bound. The hardening material parameters $A^{p}$ and $n^{p}$ appearing in the isotropic strain hardening law (6) are assumed to characterize the material behaviour in the plastic range (strain rates effects are neglected due to, as described in Section 2, the very low load cell velocity considered in the tensile tests). Their derivation from the experimental-based correlation of the mean equivalent stress versus equivalent deformation curve will be described in Section 5. Furthermore, in 
this context the effective plastic deformation rate can be also computed as $\dot{e^{p}}=\sqrt{2 / 3 \mathbf{d}^{p}: \mathbf{d}^{p}}$, where $\mathbf{d}^{\mathrm{p}}=L_{v}\left(\mathbf{e}^{\mathrm{p}}\right)$ is the plastic contribution of the rate-of-deformation tensor $\mathbf{d}$ given by $\mathbf{d}=1 / 2(\nabla \times \mathbf{v}+\mathbf{v} \times \nabla)$ where $\mathbf{v}=\dot{\mathbf{u}}$ is the velocity vector. A consequence of this model is that $\operatorname{tr}\left(\mathbf{d}^{\mathrm{p}}\right)=0$ ( $t r$ is the trace symbol) which reflects the incompressibility nature of the plastic flow that has been physically observed in many metals at moderate pressure levels (Bridgman, 1952).

The following specific free energy function $\psi=\psi\left(\mathbf{e}-\mathbf{e}^{\mathrm{p}}, \bar{e}^{p}\right)$ is proposed:

$\psi=\frac{1}{2 \rho_{0}}\left(\mathbf{e}-\mathbf{e}^{p}\right): \mathbf{C}:\left(\mathbf{e}-\mathbf{e}^{p}\right)+\frac{1}{\rho_{0}}\left(\mathbf{e}-\mathbf{e}^{p}\right): \mathbf{C}: \tau_{0}-\frac{1}{\left(n^{p}+1\right) \rho_{0}} A^{p}\left(\bar{e}_{0}^{p}+\bar{e}^{p}\right)^{n^{p}+1}+\frac{1}{\rho_{0}} C^{t h} \bar{e}^{p}+\psi_{0}$

where $\mathbf{C}$ is the isotropic elastic constitutive tensor. This last equation is a partially coupled form of defining $\psi$ which considers the density at the initial configuration according to the simplification of the Doyle-Ericksen's approach (Doyle and Ericksen, 1956). However, the elastic/plastic decomposition of $\psi$ can be considered nowadays well established since different versions of it have successfully been used in many engineering applications (see e.g. Wriggers et al., 1989; Lubliner, 1990; Simo and Armero, 1992; Armero and Simo, 1993; García Garino and Oliver, 1993; Simo, 1995; Goicolea et al., 1996; Celentano et al., 1999; Celentano, 2001 and references therein). Moreover, the additive decomposition of the Almansi strain tensor is recovered in this context through the multiplicative decomposition of the deformation gradient into elastic and plastic contributions (García Garino and Oliver, 1993). It should also be mentioned that the description of the fracture and damage phenomena are not included in the proposed specific free energy function given by equation (7).

The previous definitions allow to derive the stress-strain law (secant or hyperelastic form for the Cauchy stress tensor) and the expression of the internal dissipation which are respectively given by:

$\sigma=\frac{1}{J}\left[\mathbf{C}^{s}:\left(\mathbf{e}-\mathbf{e}^{p}-\mathbf{e}^{t h}\right)+\tau_{0}\right]$

$D_{\text {int }}=\tau: L_{v}\left(\mathbf{e}^{p}\right)+\left(C^{p}-C^{t h}\right) \dot{e}^{p} \geq 0$

The tangent form of the stress-strain law stated by equation (8) can be obtained applying standard procedures of the plasticity theory (Simo, 1995). Although this rate expression is not strictly needed within the present hyperelastic context, its derivation is particularly relevant in the computation of the stiffness matrix appearing in the finite element formulation described in Section 4. Finally, it is worth noting that the internal dissipation inequality is effectively fulfilled (i.e., both terms are separately nonnegative for every thermodynamic state) owing to the adopted definitions for the plastic evolution equations (4).

\section{FINITE ELEMENT FORMULATION}

The finite element equation derived from the model presented above are briefly described in this Section together with some important features of the numerical strategy used to solve the resulting system of discrete equations. 
Following the standard procedures within the finite element framework (Zienkiewicz and Taylor, 1989), the global discretized equilibrium equation including mass conservation can be written in matrix form for a certain time $t$ (or load level for the present quasi-static case) as:

$$
\mathbf{R}_{U} \equiv \mathbf{F}_{U}-\mathbf{F}_{\sigma}=\mathbf{0}
$$

where $\mathbf{R}_{U}$ is the residual vector, $\mathbf{F}_{\mathrm{U}}$ is the external force vector and $\mathbf{F}_{\sigma}$ denotes the internal force vector. The element expressions of these vectors can be found in Celentano (2001). It should be noted that $\mathbf{R}_{\mathrm{U}}$ is computed at the initial configuration using the well-known total Lagrangian approach (Crisfield, 1991). In this context, all the variables involved in $\mathbf{R}_{U}$ have to be transformed to the initial configuration. Moreover, a unconditionally stable generalized mid-point rule algorithm has been used to integrate the plastic rate equations presented above via a return-mapping procedure (Crisfield, 1991; Simo, 1995). On the other hand, the jacobian matrix needed in the Newton-Raphson iterative process derived by Celentano (2001) is, owing to the strong non-linearities inherent in the formulation, an approximated but numerically accurate expressions for $\mathbf{J}_{\mathrm{UU}}$ is considered where $\mathbf{K}_{\mathrm{U}}$ is the so-called stiffness matrix. In this total Lagrangian approach, the stiffness matrix consists of two terms usually denoted as the material and geometric contributions respectively related to the elastoplastic constitutive behaviour and the nonlinear effects of the adopted strain measure (Crisfield, 1991).

Although classical spatial interpolations for the displacement field have been considered in equation (10), an improved strain-displacement matrix $\overline{\mathbf{B}}$, previously proposed by Celentano (2001) and checked in problems involving moderate deformations, is also employed in this work in order to overcome the volumetric locking effect on the numerical solution when incompressible plastic flows are studied. The performance of this methodology is now tested in a large strain situation like the necking process of a cylindrical tension specimen described below. Based on the deformation gradient standard decomposition into deviatoric and volumetric parts, and assuming a selective numerical integration for the volumetric part of $\mathbf{F}$, the $\overline{\mathbf{B}}$ matrix is obtained by linearization of the Green-Lagrange strain tensor. The expressions of this matrix for the $2 \mathrm{D}$, axisymmetric and 3D cases can be found in the mentioned reference. It should mentioned that the $\overline{\mathbf{B}}$ matrix has not a sparse structure. Nevertheless, the additional computations required at element level were found not to significantly increase the CPU times in comparison with the standard sparse strain-displacement matrix. This methodology is an alternative approach to the assumed strain mixed finite element method developed by Simo and Armero (1992) in which a sparse gradient operator is obtained with the drawback of computing and storing, at element level, enhanced strain parameters defined in such context. Note that these last operations are not needed in the present $\overline{\mathbf{B}}$ algorithm and, hence, a simple computational implementation of it may be attained.

\section{EXPERIMENTAL CHARACTERIZATION AND NUMERICAL SIMULATION}

The main objective of the present analysis is to validate the predictions of the proposed formulation with the experimental data obtained in the tensile tests of SAE 1045 steel in order to achieve an adequate mechanical characterization of this material when either cylindrical or sheet specimens are considered. To this end, the experimental procedure described in Section 2 is firstly applied to cylindrical specimens. Then, the finite element formulation presented above is used to simulate the 
material behaviour during the tension deformation process (Section 5.1). Using the same material properties derived from the experiments with cylindrical specimens, the simulation of the tensile test using sheet specimens is subsequently performed (Section 5.2). An experimental validation of the numerical predictions is included for both simulations. Afterwards, a correction factor obtained from the numerical analysis with sheet specimens allows to carry out the experimental hardening characterization applied to this type of samples with the sake of checking the appropriateness of such procedure (Section $5.3)$.

\subsection{Experimental hardening characterization and simulation using cylindrical specimens}

The experimental procedure detailed in Section 2 is applied to SAE 1045 steel cylindrical specimens to characterize its plastic hardening behaviour. The correction factor $f_{B}\left(\varepsilon_{p}\right)$ proposed by Bridgman (1952) for cylindrical specimens is plotted in Fig. $5\left(\varepsilon_{p}=-2 \ln \left(D / D_{0}\right)\right.$ for this case). Fig. 6 shows the $\bar{\sigma}_{e q}$ $\varepsilon_{e q}$ average experimental data and the potential correlation $\bar{\sigma}_{e q}=A^{p} \varepsilon_{e q}^{n^{p}}$ derived from it where $A^{p}$ and $n^{p}$ are, as mentioned in Section 3, the hardening parameters. These hardening parameters are the basic data for the numerical simulation and experimental validation presented below.

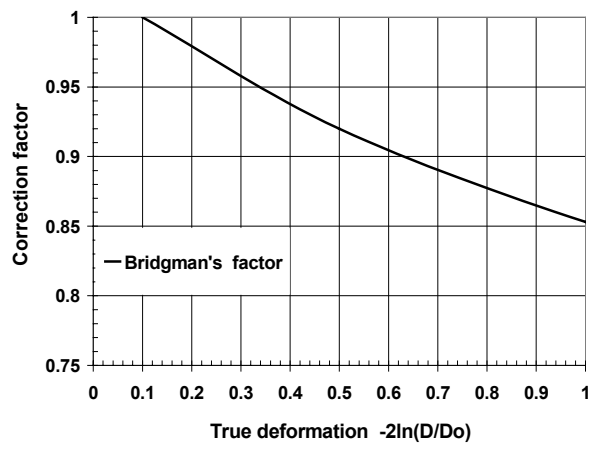

Figure 5. Analysis of a SAE 1045 steel tension specimen: Bridgman (1952) correction factor as a function of true deformation for a cylindrical sample.

The finite element formulation described in Section 4 is used to simulate the material behaviour during the tensile test. According to the experimental measurements performed during these tests, the material properties considered in the numerical analysis for SAE 1045 steel are shown in Table 3. The spatially non-uniform finite element mesh shown in Fig. 7 have been chosen in order to correctly describe the large stress and deformation gradients expected in the necking zone. Assuming axisymmetry, a fourth of the specimen is discretized with a height of $25 \mathrm{~mm}$ (half of the initial extensometer length) and a linear radius variation along the bar according to the geometry specifications depicted in Fig. 2.a where $\mathbf{U}_{\text {top }}$ denotes the axial displacement imposed at the top boundary up to a value which corresponds to the fracture elongation (see Table 2). Moreover, all the simulations are performed 
with $\mathbf{b}_{\mathrm{f}}=\mathbf{0}$ and $\tau_{0}=\mathbf{0}$.

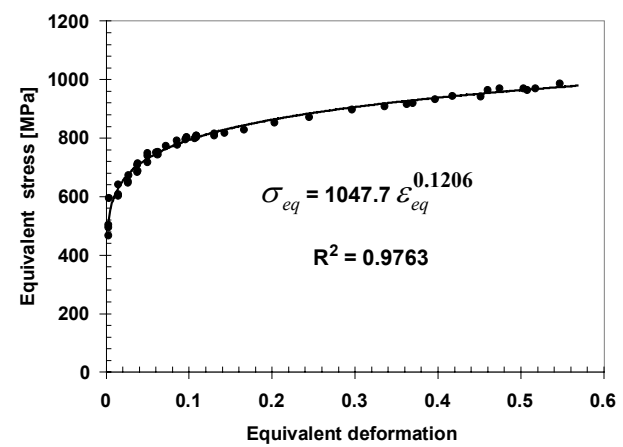

Figure 6. Analysis of a SAE 1045 steel cylindrical tension specimen: mean equivalent stress versus equivalent deformation.

Table 3. Analysis of a SAE 1045 steel tension specimen: material properties considered in the numerical simulations.

\begin{tabular}{|l||c||}
\hline Young's modulus $E$ & $222000[\mathrm{MPa}]$ \\
\hline \hline Poisson's ratio $v$ & 0.30 \\
\hline \hline Yield strength $C^{\text {th }}$ & $450[\mathrm{MPa}]$ \\
\hline \hline Hardening coefficient $A^{\mathrm{p}}$ & 1047.7 \\
\hline \hline Hardening exponent $n^{\mathrm{p}}$ & 0.1206 \\
\hline
\end{tabular}

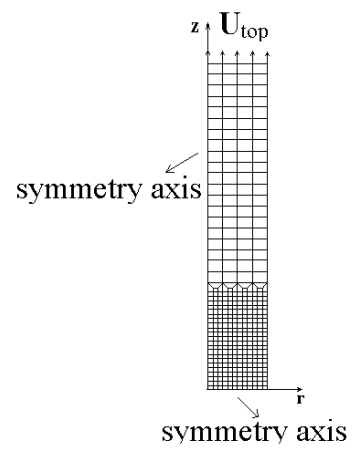

Figure 7. Analysis of a SAE 1045 steel cylindrical tension specimen: axisymmetric finite element mesh used in the simulation (mesh composed of 360 four-noded isoparametric elements). 
Fig. 8 shows the engineering stress-strain relationship and some results at the section undergoing extreme necking: the radii relation versus the elongation in the necking zone together with the load and mean true axial stress both against the logarithmic deformation. An overall good agreement between the numerical predictions and the average experimental values can be observed in these curves. The discrepancies appearing in the engineering stress-strain curve can be mainly attributed to the inaccuracy of the potential correlation at the beginning of the plastic region where no hardening is produced.
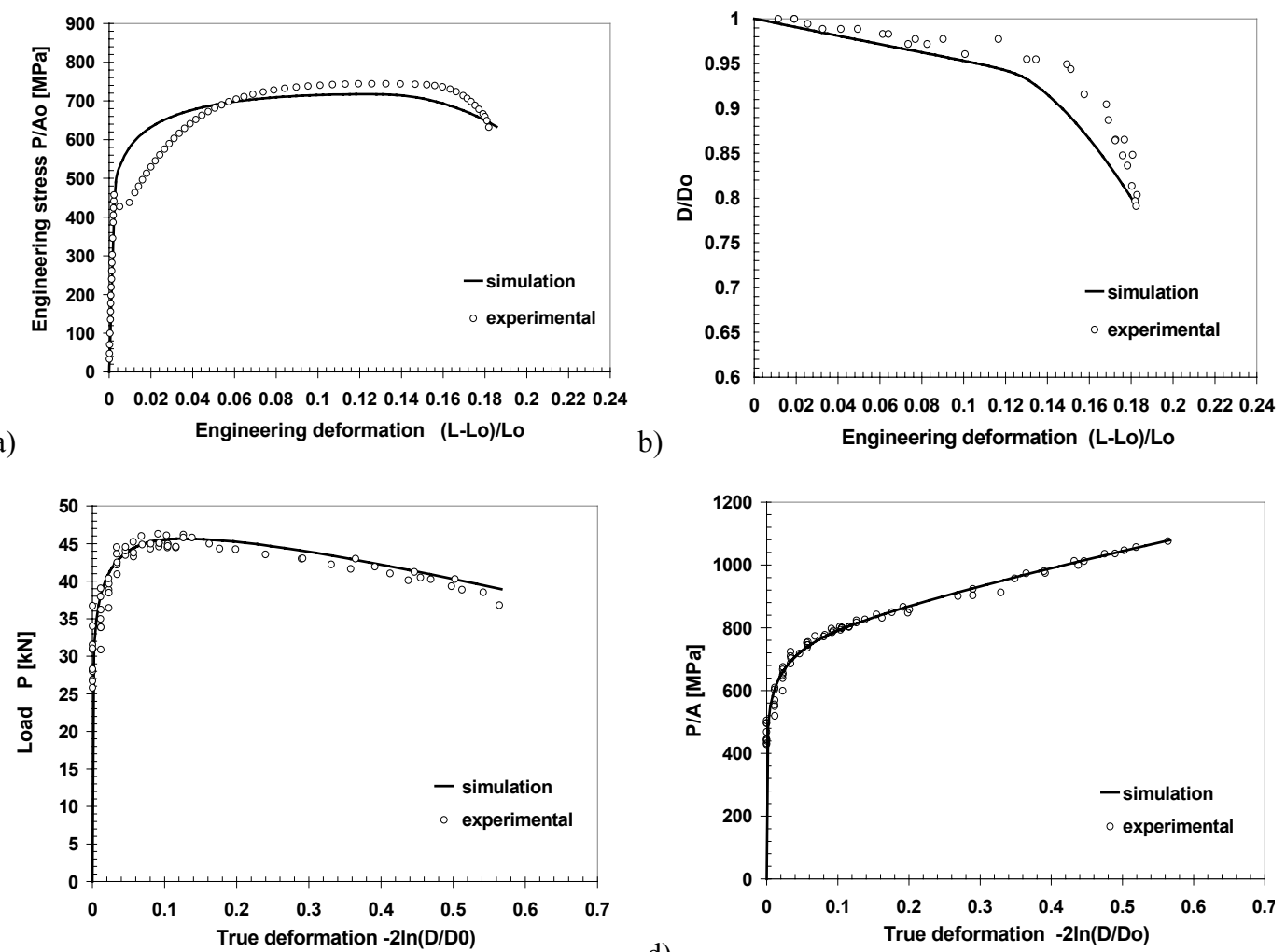

c)

d)

Figure 8. Analysis of a SAE 1045 steel cylindrical tension specimen. a) Engineering stress-strain relationship. Results at the section undergoing extreme necking: b) ratio of current to initial diameter versus axial elongation, c) load versus true deformation and d) mean true axial stress versus true deformation.

The experimentally measured load decreases from an elongation of $11.5 \%$ or, equivalently, to a logarithmic deformation $\varepsilon_{p}$ of $11.2 \%$ onwards (the corresponding deformations provided by the 
simulation are $12.4 \%$ and $11.7 \%$, respectively). However, the mean true axial stress continues increasing until the fracture stage where a big amount of plastic hardening can be appreciated. This fact confirms that a geometrical instability occurs (instead of a constitutive instability) since, as already commented in Section 2, the effect on the stress caused by the reduction of the transversal area at the necking zone predominates over the material hardening. At high level of deformations, the regions of the specimen outside the necking zone are being elastically unloaded. Moreover, note that the wellknown simplified relationship (Dieter, 1988), stating that the related logarithmic deformation at the point of maximum load has to be equal to the hardening exponent, is approximately verified (see Table 3 and Figure 6).

The numerical predictions for the ratio of current to initial diameter in terms of the elongation starts with a linear relationship, reflecting uniform distributions of stresses and strains, which presents an approximate slope of 0.5 due to the incompressibility nature of the plastic flow. The same situation is kept up to an elongation of $12.5 \%$ which corresponds, as mentioned above, to the point of maximum load. Afterwards, a sudden reduction of the diameter takes place causing the necking formation and, hence, non-homogeneous stress and strain distributions along the specimen. As can be seen, the numerical results fit reasonably well the experimental ones during the whole test even though the inherent difficulty associated with the measurement of the diameter at the neck.

Fig. 9 compares the correction factor in terms of true deformation proposed by Bridgman (1952) with that obtained through the numerical simulation as the quotient between the average equivalent stress at the neck and the mean true axial stress $P / A$. It is observed that both curves present practically the same response (the maximum discrepancy existing within the range of deformation $0 \%-57 \%$ experienced by the material as shown in Fig. 8 is less than $1 \%$ ). A uniform stress distribution, expressed by the condition $f_{B} \approx 1$, is obtained for $\varepsilon_{p} \leq 0.1$. For larger deformations, triaxial stresses are developed and, therefore, the effect of the correction factor on $P / A$ becomes relevant.

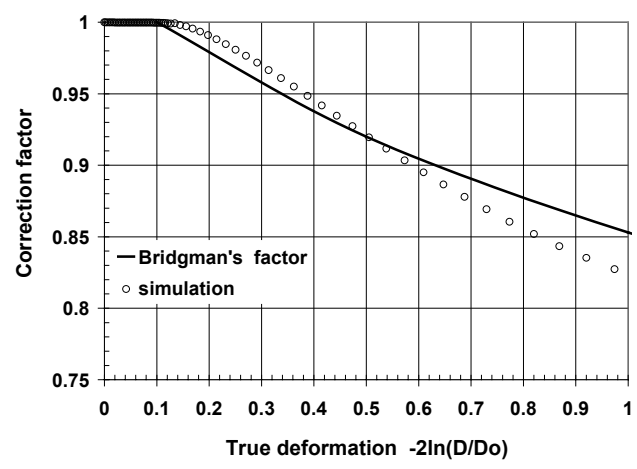

Figure 9. Analysis of a SAE 1045 steel tension specimen: simulated correction factor as a function of true deformation for a cylindrical sample. 
Different stress components (hoop, radial, axial and shear components of $\sigma$ together with the pressure $p=1 / 3 \operatorname{tr}(\sigma)$ and the equivalent stress $\sigma_{\text {eq }}$ given by $\left.\sqrt{3 J_{2}}\right)$ and effective plastic deformation contours at the end of the analysis (fracture stage) can be found in Fig. 10. Non-uniform distributions are clearly obtained due to the complex deformation pattern of the neck. As expected, the maximum values of $\sigma_{\text {eq }}$ and consequently of $\bar{e}^{p}$ are concentrated in the neck. Note that values around 0.10 of effective plastic deformation mainly found at the rear part of the specimen indicate the level of uniform deformation experienced until the maximum load is reached (see Fig. 8). The neck formation even induces the development of low pressures at the center of the bar (this fact has also been pointed out by Armero and Simo (1993) and Goicolea et al. (1996) in the tension analysis of other materials). Furthermore, some assumptions considered in the analytical study of Bridgman (1952) at the neck are ratified by the simulation, e.g., $\sigma_{\mathrm{eq}}$ and $\bar{e}^{p}$ are approximately constant, $\sigma_{\mathrm{rr}}$ and $\sigma_{\theta \theta}$ present a strong variation but the condition $\sigma_{\mathrm{rr}} \approx \sigma_{\theta \theta}$ is fullfiled, $\sigma_{\mathrm{zz}} \approx \sigma_{\mathrm{eq}}+\sigma_{\mathrm{rr}}>\sigma_{\mathrm{eq}}$ that explains the need to correct the stress distribution, $p \approx \sigma_{\mathrm{eq}} / 3+\sigma_{\mathrm{rr}}, e_{z z} \approx-2 e_{r r}$ up to the maximum load and $e_{r r} \approx e_{\theta \theta}$ during the whole test.

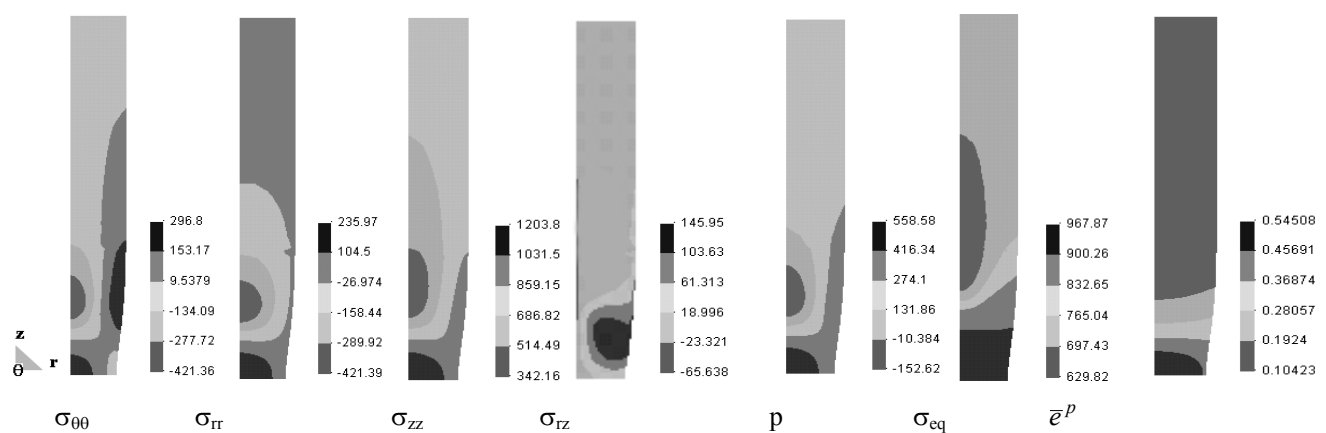

Figure 10. Analysis of a SAE 1045 steel cylindrical tension specimen: stress (in MPa) and effective plastic deformation contours at the end of the simulation corresponding to the fracture stage for an elongation of $18.5 \%$.

\subsection{Simulation using sheet specimens}

The previously described finite element formulation is also used to simulate the material response during the tensile test using sheet specimens considering the same properties derived from the experiments with cylindrical samples (see Table 3). The 3D finite element mesh is shown in Fig. 11 where, due to symmetry, only one eighth is studied (note that this mesh presents a similar element densification as that shown in Fig. 7). Fitting the specifications of Fig. 2.b, the neck in the middle of the specimen is triggered by assuming a linear width variation along its length. As in the previous analysis, $\mathbf{U}_{\text {top }}$ denotes the prescribed axial displacement imposed at the top boundary up to fracture.

The engineering stress-strain relationship together with some results at the necking zone (width and 
thickness ratios versus the elongation and the load and mean true axial stress both against the logarithmic deformation) are plotted in Fig. 12. Once more, an overall good agreement between the numerical predictions and the average experimental values can be observed in these curves. The discrepancies appearing in the engineering stress-strain curve can be also attributed to the inaccuracy of the potential correlation at the beginning of the plastic region where little hardening is produced.

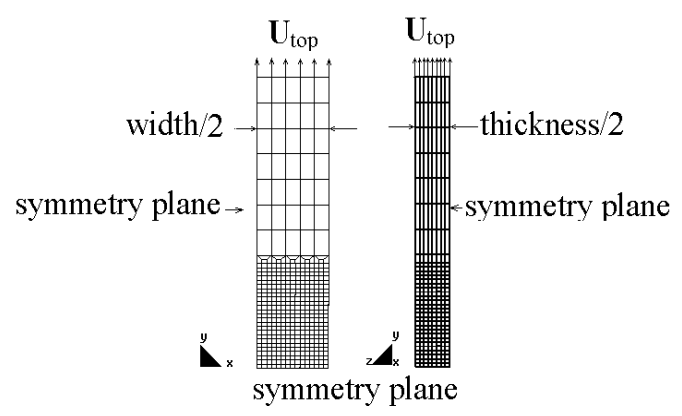

Figure 11. Analysis of a SAE 1045 steel sheet tension specimen: 3D finite element mesh used in the simulation (mesh composed of 3440 eight-noded isoparametric elements).

Although some small discrepancies in the load and mean true axial stress appear at higher levels of deformation, it should be noted that such differences are approximately bounded within the experimental uncertainty range.

The experimentally measured load decreases from an elongation of $12.5 \%$ or, equivalently, to a logarithmic deformation $\varepsilon_{p}$ of $11.8 \%$ onwards (the corresponding deformations provided by the simulation are $13.0 \%$ and $12.2 \%$, respectively). A geometrical instability caused by the necking formation is also observed for this case since the mean true axial stress continues increasing until the fracture stage.

The numerical predictions for the $w / w_{o}$ and $t / t_{o}$ ratios match each other at the beginning of the test since a uniaxial stress is achieved for a low level of elongation in the range $0 \%-13.0 \%$. Once the neck is formed, both curves progressively differ where, as in the experiments, the reduction in $t / t_{o}$ is stronger than that of $w / w_{o}$.

The six components of $\sigma$, the pressure $p$, the equivalent stress $\sigma_{\mathrm{eq}}$ and effective plastic deformation contours at the fracture stage are plotted in Fig. 13. Once again, non-uniform distributions are clearly obtained due to the complex deformation pattern of the neck where, as expected, the maximum values of $\sigma_{\mathrm{eq}}$ and consequently of $\bar{e}^{p}$ are concentrated in the neck. In particular, note that a very strong variation of the effective plastic deformation is found at the neck. Only positive pressures are obtained for this case.

Fig. 14 shows the correction factor in terms of true deformation obtained from the simulation. This curve is also presented in Table 4. For a given value of logarithmic strain, note that the effect of the 
correction factor is larger for sheet specimens than that for cylindrical ones.

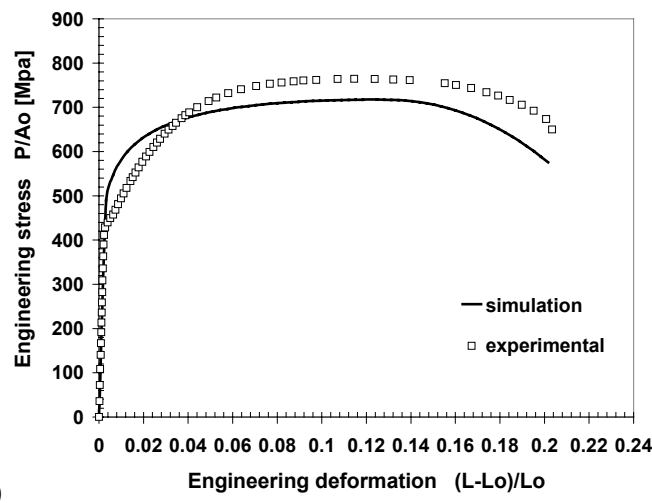

a)

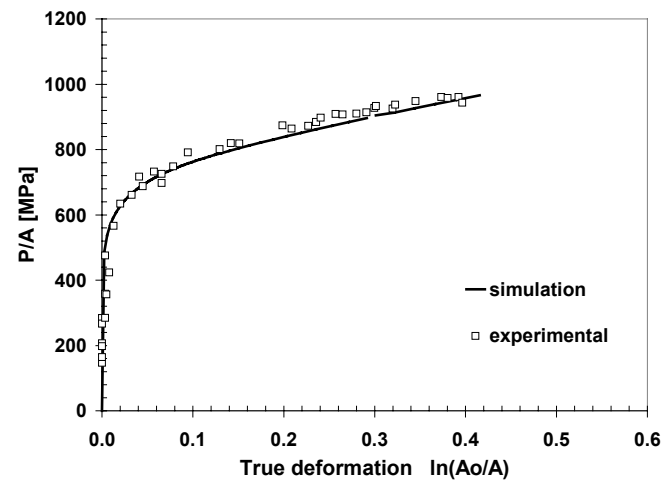

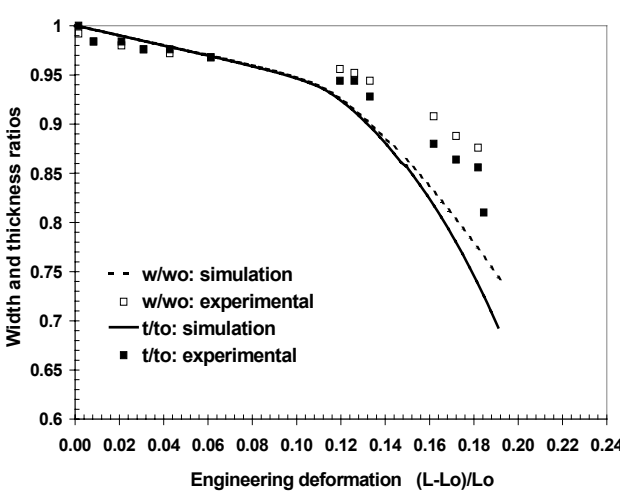

b)

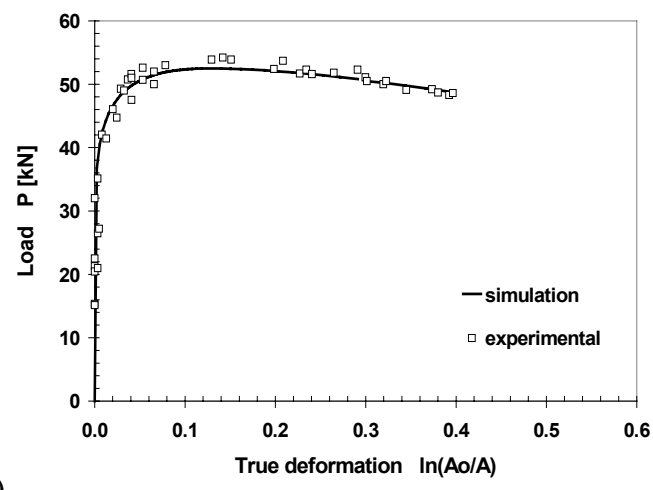

Figure 12. Analysis of a SAE 1045 steel sheet tension specimen. a) Engineering stress-strain relationship. Results at the section undergoing extreme necking: b) ratio of current to initial width and thickness (mean values along thickness and width, respectively) versus axial elongation, c) load versus true deformation and d) mean true axial stress versus true deformation.

\subsection{Experimental hardening characterization using sheet specimens}

The consideration of the proposed correction factor of Fig. 14 allows the derivation of the experimental $\bar{\sigma}_{e q}-\varepsilon_{e q}$ curve for sheet specimens. The average measured data together with the corresponding potential correlation $\bar{\sigma}_{e q}=A^{p} \varepsilon_{e q}^{n^{p}}$ are both shown in Fig. 15. As can be appreciated, these hardening parameters are practically the same to those of Fig. 6 for cylindrical specimens. This 
proposed methodology shows, therefore, that an adequate characterization of the material response can be also achieved for sheet samples.

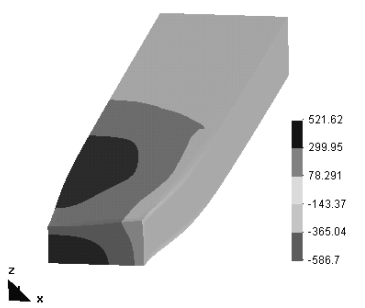

$\sigma_{\mathrm{xx}}$

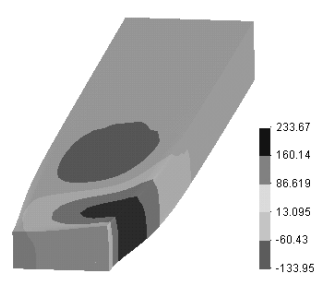

$\sigma_{\mathrm{zz}}$

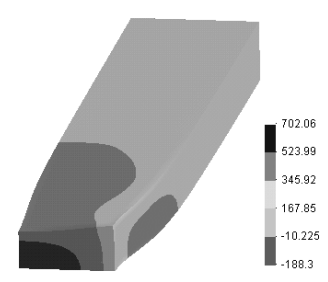

$\mathrm{p}$

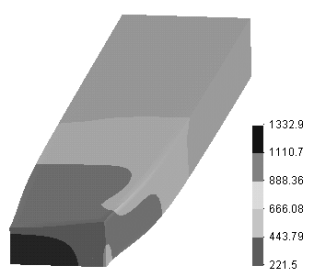

$\sigma_{\mathrm{yy}}$

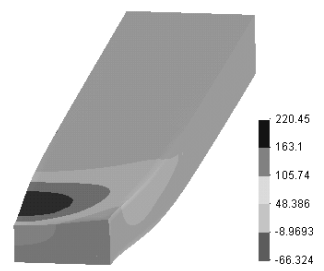

$\sigma_{\mathrm{xz}}$

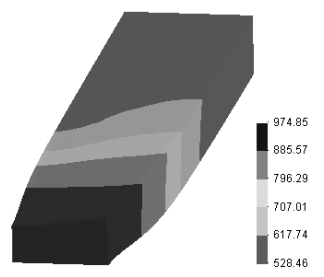

$\sigma_{\text {eq }}$

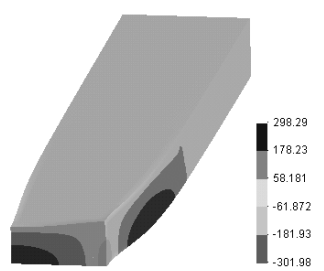

$\sigma_{\mathrm{xy}}$

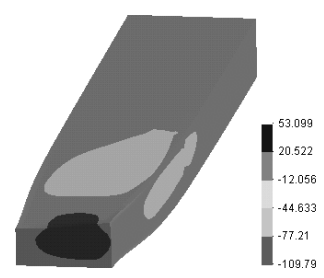

$\sigma_{\mathrm{yz}}$

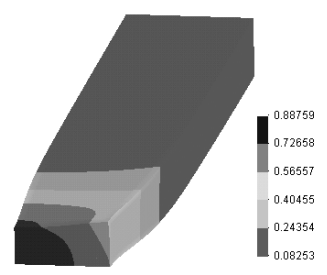

$\bar{e}^{p}$

Figure 13. Analysis of a SAE 1045 steel cylindrical tension specimen: stress (in MPa) and effective plastic deformation contours at the end of the simulation corresponding to the fracture stage for an elongation of $20.0 \%$. 


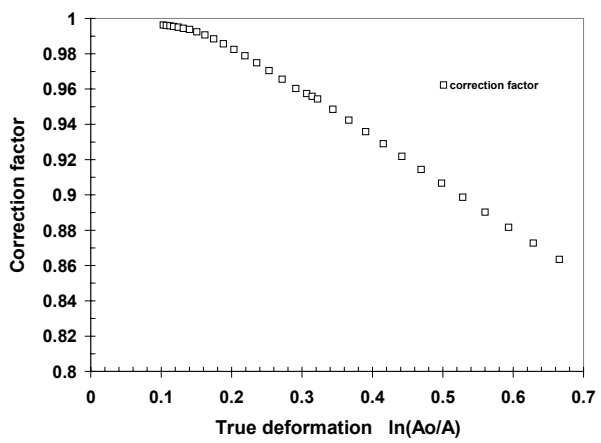

Figure 14. Analysis of a SAE 1045 steel tension specimen: simulated correction factor as a function of true deformation for a sheet sample.

Table 4. Analysis of a SAE 1045 steel tension specimen: correction factor as a function of true strain.

\begin{tabular}{|c||c||c|}
\hline \multirow{2}{*}{\multicolumn{1}{|c||}{$\ln (\mathbf{A o} / \mathbf{A})$}} & \multicolumn{2}{c|}{ Correction factor } \\
\cline { 2 - 3 } & Cylindrical sample & Sheet sample \\
\hline \hline $\mathbf{0 . 1 0}$ & 1.000 & 1.000 \\
\hline \hline $\mathbf{0 . 2 0}$ & 0.978 & 0.976 \\
\hline \hline $\mathbf{0 . 3 0}$ & 0.957 & 0.955 \\
\hline $\mathbf{0 . 4 0}$ & 0.938 & 0.933 \\
\hline \hline $\mathbf{0 . 5 0}$ & 0.921 & 0.909 \\
\hline \hline $\mathbf{0 . 6 0}$ & 0.905 & 0.884 \\
\hline $\mathbf{0 . 7 0}$ & 0.890 & 0.858 \\
\hline \hline $\mathbf{0 . 8 0}$ & $\mathbf{0 . 8 7 6}$ & $\mathbf{0 . 8 3 0}$ \\
\hline \hline $\mathbf{0 . 9 0}$ & $\mathbf{0 . 8 6 3}$ & $\mathbf{0 . 8 0 0}$ \\
\hline \hline $\mathbf{1 . 0 0}$ & $\mathbf{0 . 8 5 1}$ & $\mathbf{0 . 7 6 9}$ \\
\hline
\end{tabular}

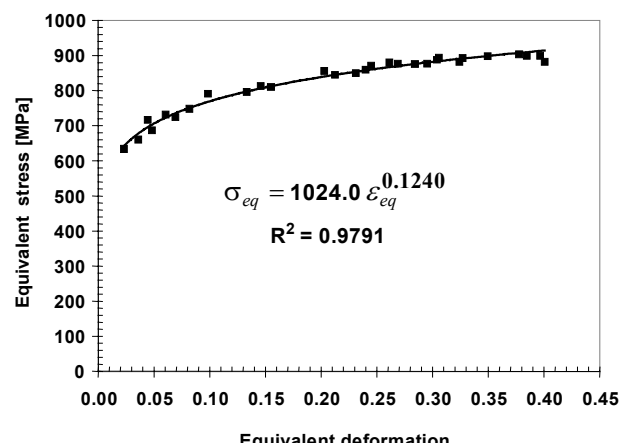

Figure 15. Analysis of a SAE 1045 steel sheet tension specimen: mean equivalent stress versus equivalent deformation. 


\section{CONCLUSIONS}

Experimental and numerical analyses of the mechanical behaviour occurring in cylindrical and sheet specimens during the standard tensile test applied to SAE1045 steel have been presented. A characterization of the material response using cylindrical specimens has been firstly performed in order to obtain the stress-strain curve and the diameter evolution at the neck which allow, in turn, to derive the elastic and hardening by means of a well established methodology. Then, a finite element large strain elastoplasticity-based formulation has been proposed and used to simulate the tensile deformation process in such type of samples. Moreover, these material properties have been considered in the subsequent numerical analysis of the tensile test using sheet specimens. The results provided by both simulations have been satisfactorily validated with experimental data. Afterwards, a successful experimental hardening characterization carried out for sheet specimens using a correction factor deduced from the simulation has been performed. Finally, the proposed methodology has enabled to achieve an adequate description of the mechanical response of the material during the tensile test when using either cylindrical or sheet specimens.

\section{Acknowledgments}

The supports provided by the Chilean Council of Research and Technology CONICYT (FONDECYT Project $\mathrm{N}^{\mathrm{o}}$ 1020026) and the Department of Technological and Scientific Research at the University of Santiago de Chile (DICYT-USACH) are gratefully acknowledged. The authors wish to express their appreciation to the Aeronautical Technical Academy at Santiago de Chile for the provision of experimental facilities.

\section{REFERENCES}

Annual Book of ASTM Standards (1988). Section 3: Metals Test Methods and Analytical Procedures.

Armero F. and Simo J. (1993) A priori stability estimates and unconditionally stable product formula algorithms for non-linear coupled thermoplasticity, International Journal of Plasticity 9, 149-182.

Bridgman P. (1952) Studies in Large Plastic and Fracture, McGraw-Hill Book Company, London.

Celentano D., Gunasegaram D. and Nguyen T. (1999) A thermomechanical model for the analysis of light alloy solidification in a composite mould, International Journal of Solids and Structures, Vol. 36, 2341-2378.

Celentano D. (2001) A large strain thermoviscoplastic formulation for solidification of S.G. cast iron in a green sand mould, International Journal of Plasticity 17, 1623-1658.

Coleman B. and Gurtin M. (1967) Thermodynamics with internal state variables, The Journal of Chemical Physics 47 (2), 597-613.

Crisfield M. (1991) Non-linear Finite Element Analysis of Solids and Structures, Vols. 1 and 2. John Wiley \& Sons, Chichester.

Dieter G. (1988) Mechanical Metallurgy - SI Metric Edition, McGraw-Hill Book Company, London. 
Doyle, T. and Ericksen, J. (1956) Nonlinear elasticity, Advances in Applied Mechanics 4.

García Garino C. and Oliver J. (1993) A numerical model for elastoplastic large strain problems. Fundamentals and applications, Proceedings of COMPLAS III, 117.

Goicolea J., Gabaldón F. and García Garino C. (1996) Analysis of the tensile test using hypo and hyperlastic models (in Spanish), Proceedings of the III Congress on Numerical Methods in Engineering, 875-885.

Ling L. (1996) Uniaxial true stress-strain after necking, AMP Journal of Technology, Vol. 5, 37-48.

Lubliner J. (1990) Plasticity Theory, Macmillan Publishing.

Malvern L. (1969) Introduction to the Mechanics of a Continuous Medium, Prentice-Hall, Englewood Cliffs.

Nádai A. (1950) Theory of Flow and Fracture of Solids, McGraw-Hill Book Company, London.

Simo J. and Armero F. (1992) Geometrically non-linear enhanced strain mixed methods and the method of incompatible modes, International Journal for Numerical Methods in Engineering, Vol. 33, 14131449.

Simo J. (1995) Topics on the Numerical Analysis and Simulation of Plasticity, Handbook of Numerical Analysis, Vol. III, Elsevier Science Publishers.

Tvergaard V. (1993) Necking in tensile bars with rectangular cross-section, Computer Methods in Applied Mechanics and Engineering, Vol. 103, 273-290.

Wriggers P., Miehe C., Kleiber M. and Simo J. (1989) On the coupled thermo-mechanical treatment of necking problems via finite-element-method, Proceedings of COMPLAS II, 527-542.

Zienkiewicz, O. and Taylor, R. (1989) The Finite Element Method, $4^{\text {th }}$ Edition, Vols. 1 and 2. McGrawHill, London. 